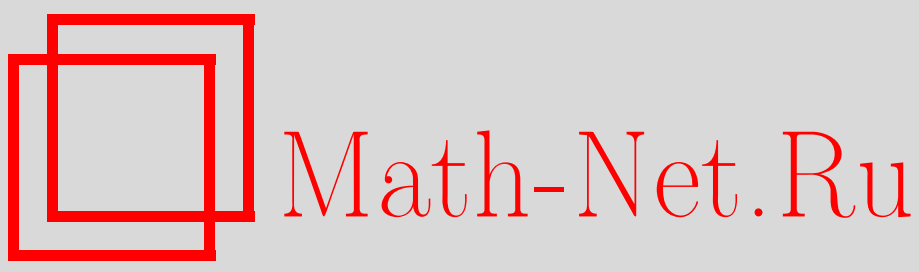

M. V. Zubkov, A. N. Frolov, Spectral universality of linear orders with one binary relation, Sibirsk. Mat. Zh., 2020, Volume 61, Number 3, 587-593

DOI: https://doi.org/10.33048/smzh.2020.61.307

Use of the all-Russian mathematical portal Math-Net.Ru implies that you have read and agreed to these terms of use http://www . mathnet.ru/eng/agreement

Download details:

IP: 54.157 .27 .8

April 26, 2023, 10:05:43

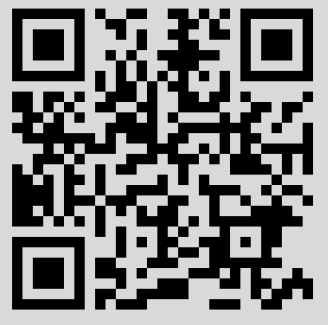


Сибирский математический журнал Май-июнь, 2020. Том 61, № 3

УДК $510.53+512.562$

\title{
СПЕКТРАЛЬНАЯ УНИВЕРСАЛЬНОСТЬ ЛИНЕЙНЫХ ПОРЯДКОВ С ОДНИМ БИНАРНЫМ ОТНОШЕНИЕМ
}

\author{
М. В. Зубков, А. Н. Фролов
}

\begin{abstract}
Аннотация. Показана спектральная универсальность класса структур, представляющих собой линейные порядки с дополнительным бинарным отношением и, следовательно, $n$-арным отношением для любого наперед заданного $n \geq 2$. Для этого исследуется категория таких структур. Это позволяет, используя понятие вычислимого функтора, исследуемое в недавних работах других авторов, получить ряд прочих алгоритмических свойств этой категории.
\end{abstract}

DOI 10.33048/smzh.2020.61.307

Ключевые слова: вычислимые линейные порядки, спектральная универсальность, бинарные отношения.

\section{1. Введение}

Множество тьюринговых степеней, относительно которых данная алгебраическая структура $\mathscr{A}$ является вычислимо представимой, называется спектром степеней данной структуры и обозначается через $\operatorname{dgSp}(\mathscr{A})$. Вопрос описания спектров степеней алгебраических структур является одним из фундаментальных вопросов теории вычислимых структур и их моделей. А. Слинько, Д. Хиршфельдт, Б. Хусаинов, Р. Шор [1] назвали класс структур спектрально универсальным, если любой возможный спектр степеней алгебраической структуры реализуется спектром степеней структуры из данного класса. Другими словами, класс структур $\mathscr{P}$ называется спектрально универсальным, если для любой алгебраической структуры $\mathscr{A}$ существует структура $\mathscr{B} \in \mathscr{P}$ такая, что $\operatorname{dgSp}(\mathscr{B})=\operatorname{dgSp}(\mathscr{A})$.

Ими же была установлена спектральная универсальность ряда классических классов алгебраических структур, например, таких классов, как неориентированные графы, решетки, коммутативные полугруппы и другие. Ряд других примеров, в том числе и новых, а также обзор результатов, описывающих спектры степеней различных структур, можно найти в недавней работе И. Калимуллина, В. Селиванова и А. Фролова [2].

В работе В. Харизановой и Миллера [3] показывается, что любой возможный спектр степеней реализуется спектром некоторого унарного (и, следовательно, $n$-арного для любого наперед заданного $n \geq 1$ ) отношения на вычислимом случайном графе. В этом случае также говорят, что это отношение спектрально универсально. П. Е. Алаев (не опубликовано, см., например, [2])

Работа М. В. Зубкова поддержана грантом РФФИ №18-31-00174, работа А. Н. Фролова - грантом Президента РФ МД-2721.2019.1.

(c) 2020 Зубков М. В., Фролов А. Н. 
доказал такой же результат для унарного отношения на вычислимой булевой алгебре $\mathscr{B}_{\omega}$. Нетрудной модификацией этот результат можно перенести на вычислимую безатомную булеву алгебру и показать, что каждый спектр степеней алгебраической структуры реализуется спектром унарного отношения вычислимой безатомной булевой алгебры.

Класс счетных линейных порядков один из наиболее сложных с точки зрения описания спектров степеней всех представителей этого класса. Он не спектрально универсальный. Это следует, например, из того, что спектр степеней линейного порядка в отличие от графов может образовывать верхний конус степеней тогда и только тогда, когда он содержит вычислимую степень (Рихтер [4]). Также до сих пор неизвестно, существует ли линейный порядок, спектр степеней которого содержит в точности все ненулевые степени (некоторые частичные продвижения можно найти в [5-7]).

В. Харизанова и Миллер [3] доказали результат Рихтер для унарного отношения на произвольном линейном порядке. Таким образом, класс структур, являющихся линейными порядками, сигнатура которых обогащена унарным отношением, спектрально универсальным не является.

В данной работе доказано, что бинарное (и, следовательно, $n$-арное для любого $n \geq 2$ ) отношение на $\mathbb{Q}$ (естественное упорядочение множества рациональных чисел) спектрально универсально. А именно, показано, что для любого графа существует бинарное отношение на $\mathbb{Q}$, спектр которого совпадает со спектром степеней графа.

\section{2. Основной результат}

Определение бинарного отношения на линейном порядке фактически означает определение на нем графа. Возможно, читателю будет удобнее представить такую структуру иначе, а именно как упорядоченный граф. Другими словами, рассмотрим категорию (обозначим ее через LBin) структур вида $\mathscr{A}=\{\omega ;<, E\}$, где $\mathscr{A}=\{\omega ;<\}-$ линейный порядок, а $\mathscr{A}=\{\omega ; E\}-$ неориентированный граф без петель, морфизмами здесь являются изоморфные вложения. Обозначим категорию, объектами которой являются неориентированные графы без петель, а морфизмами - изоморфные вложения графов, через Graph.

В [8] введено понятие вычислимого функтора, которое позволяет транслировать алгоритмические свойства, например спектральную универсальность, категоричность и т. п., из одной категории структур в другую.

ОПРЕДЕЛЕниЕ. Пусть $\mathfrak{C}$ и $\mathfrak{D}$ - категории структур с основным множеством $\omega$, для которых морфизмы из структуры $\mathscr{S}$ в структуру $\mathscr{T}$ суть отображения из основного множества $\omega$ структуры $\mathscr{S}$ в основное множество $\omega$ структуры $\mathscr{T}$. Вычислимым называется функтор $\mathscr{F}: \mathfrak{C} \rightarrow \mathfrak{D}$ такой, что существуют тьюринговы функционалы $\Phi$ и $\Phi_{*}$ со следующими свойствами:

- для каждой структуры $\mathscr{S} \in \mathfrak{C}$ функция $\Phi^{\mathscr{S}}$ вычисляет структуру (атомную диаграмму) $\mathscr{F}(\mathscr{S})$;

- для каждого морфизма $g: \mathscr{S} \rightarrow \mathscr{T}$ в $\mathfrak{C}$ имеем $\Phi_{*}^{\mathscr{S} \oplus g \oplus \mathscr{T}}=\mathscr{F}(g)$ в $\mathfrak{D}$.

Кроме того, в [9] доказано, что существование вычислимого функтора с некоторыми дополнительными условиями эквивалентно би-интерпретируемости структур.

Назовем вычислимый функтор $\mathscr{F}: \mathfrak{C} \rightarrow \mathfrak{D}$ вычислимо обратимым, если существует вычислимый функтор $\mathscr{F}^{-1}: \operatorname{rng}(\mathscr{F}) \rightarrow \mathfrak{C}$ такой, что для любой $\mathscr{A} \in \mathfrak{C}$ существуют морфизмы $g_{1}: \mathscr{F}^{-1}(\mathscr{F}(\mathscr{A})) \rightarrow \mathscr{A}$ и $g_{2}: \mathscr{A} \rightarrow \mathscr{F}^{-1}(\mathscr{F}(\mathscr{A}))$. 
Теорема. Существует инъективный вычислимо обратимый вычислимый функтор

$$
\mathscr{F}: \text { Graph } \rightarrow \text { LBin. }
$$

ДокАЗАТЕЛЬСтво. Прежде чем описать полное доказательство теоремы, сначала приведем идею доказательства спектральной универсальности частично-упорядоченного множества (ч.у.м.), идею кодирования графа в ч.у.м. с сохранением спектра проста. Объявляем все четные числа не сравнимыми друг с другом и сопоставляем их с элементами графа $2 x \mapsto x$. Берем наименьшее еще не использованное нечетное число $t$. Если между элементами графа $a$ и $b$ имеется ребро, то объявляем $t=2 a \vee 2 b$, если между ними ребра нет, то $t=2 a \wedge 2 b$. Совпадение спектров графа и построенного ч.у.м. очевидно.

Прямая адаптация этой идеи для рассматриваемой категории структур невозможна. Пусть дан граф $\mathscr{G}=\left\{\omega ; E_{G}\right\}$. Предположим, что так же, как и выше, при построении линейно упорядоченного графа $\mathscr{A}(\mathscr{G})=\{A ;<, E\}$ каждому элементу графа $x$ ставим в соответствие некоторый элемент $y$. Тогда после линейного упорядочивания в $\mathscr{A}$ всех образов нельзя обеспечить выполнение следующего основного свойства: если $\mathscr{G}_{1} \cong \mathscr{G}_{2}$, то $\mathscr{A}\left(\mathscr{G}_{1}\right) \cong \mathscr{A}\left(\mathscr{G}_{2}\right)$. Такая же проблема возникает при линейном упорядочивании верхних и нижних граней из предыдущего описания.

Для преодоления этой проблемы применим следующий прием (как для образов элементов графа $\mathscr{G}$, так и для образов ребер, т. е. для верхних и нижних граней). Каждому элементу графа ставим в соответствие бесконечное множество элементов структуры $\mathscr{A}$. Эти множества образов для разных элементов графа плотно перемешиваем в линейном порядке. Чтобы не возникло путаницы между разными образами, соединяем попарно ребром все элементы $\mathscr{A}$, соответствующие одному элементу $\mathscr{G}$. Аналогичную процедуру применяем для элементов, соответствующих верхним и нижним граням из предыдущего описания. Такое адаптирование предыдущей идеи позволяет организовать кодирование графа в бинарное отношение линейного порядка с сохранением спектра.

Перейдем теперь к описанию полного доказательства. Сначала произвольный граф $\mathscr{G}$ превратим в граф $\mathscr{G}^{\prime}$, имеющий бесконечное множество ребер и бесконечное множество пар не соседних элементов. Каждой вершине графа $\mathscr{G}$ в $\mathscr{G}^{\prime}$ будет соответствовать подграф, изоморфный $K_{3}$ (т. е. полный граф с тремя вершинами). Будем обозначать их через $\mathscr{G}_{x}^{\prime}$. Если вершины $x$ и $y$ соединены ребром в $\mathscr{G}$, то каждая вершина $\mathscr{G}_{x}^{\prime}$ соединена с каждой вершиной $\mathscr{G}_{y}^{\prime}$ уникальным путем длины 2. В противном случае каждая вершина $\mathscr{G}_{x}^{\prime}$ соединена с каждой вершиной $\mathscr{G}_{y}^{\prime}$ уникальным путем длины 3 . Нетрудно видеть, что данное соответствие задает инъективный вычислимо обратимый вычислимый функтор из категории Graph в категорию Graph. При этом образ всегда имеет бесконечное множество ребер и бесконечное множество пар не соседних элементов. Далее при построении вычислимого функтора $\mathscr{F}$ из категории счетных графов $\mathscr{G}=\left\{\omega ; E_{G}\right\}$ в категорию счетных графов с линейным порядком $\mathscr{A}=\{\omega ;<, E\}$ будем предполагать, что граф обладает данным свойством. Искомый функтор будет являться композицией двух построенных.

Построение функтора. Разобьем $\mathbb{Q}$ при помощи двух элементов $q_{0}$ и $q_{1}$ на три непересекающихся интервала $I_{1}<q_{0}<I_{2}<q_{1}<I_{3}$. Разобьем каждый интервал $I_{i}, 1 \leq i \leq 3$, на счетное равномерно вычислимое семейство подмножеств $I_{i, j}$ так, что

1) $I_{i, j} \cap I_{i, k}=\varnothing$ при $j \neq k$; 
2) $I_{i}=\bigcup_{j \in \omega} I_{i, j}$

3) $\left(\forall x<y ; x, y \in I_{i, j}\right)(\forall k)\left(\exists z \in I_{i, k}\right)[x<z<k]$.

Каждое множество $I_{2, j}$ будет кодировать одну вершину графа $\mathscr{G}$. Для каждого $j$ и $x, y \in I_{2, j}$ полагаем $\{x, y\} \in E$.

Множества $I_{3, j}$ будем использовать для кодирования ребер графа $\mathscr{G}$. Пусть $\nu: E_{G} \rightarrow \omega-$ некоторая эффективная биективная нумерация ребер графа $\mathscr{G}=\left\{\omega ; E_{G}\right\}$. Если $E_{G}\left(v_{1}, v_{2}\right)$, то для всех $x \in I_{2, v_{1}}, y \in I_{2, v_{2}}, z \in I_{3, \nu\left(\left\{v_{1}, v_{2}\right\}\right)}$ полагаем $\{x, z\} \in E$ и $\{z, y\} \in E$.

Осталось закодировать случай, когда между вершинами графа $\mathscr{G}$ отсутствуют ребра. Для этого воспользуемся семейством $I_{1, j}$. Пусть $\mu: \omega^{2} \backslash E_{G} \rightarrow \omega-$ некоторая эффективная биективная нумерация пар не соседних вершин графа $\mathscr{G}=\left\{\omega ; E_{G}\right\}$. Если $\neg E_{G}\left(v_{1}, v_{2}\right)$, то для всех $x \in I_{2, v_{1}}, y \in I_{2, v_{2}}, z \in I_{1, \mu}\left(v_{1}, v_{2}\right)$ полагаем $\{x, z\} \in E$ и $\{z, y\} \in E$.

ЭФФЕКТИвНОЕ ОБРАщЕНИЕ $\mathscr{F}$. Опишем, как эффективно восстановить граф $\mathscr{G}=\left\{\omega ; E_{G}\right\}$ по структуре $\mathscr{A}=\{A ;<, E\}=\mathscr{F}(\mathscr{G})$. Соответствующий функтор обозначим через $\mathscr{F}^{-1}$. Отметим, что два элемента $x, y \in I_{2}$ соответствуют различным вершинам графа $\mathscr{G}$ тогда и только тогда, когда они не являются соседними в $\mathscr{A}$.

Пусть $x, y \in \mathscr{A}$ соответствуют различным вершинам $v_{1}, v_{2}$ графа $\mathscr{G}$. Элементы $v_{1}$ и $v_{2}$ являются соседними в графе $\mathscr{G}$ тогда и только тогда, когда в $\mathscr{A}$ между $x$ и $y$ есть путь $x-t-y$ такой, что $t>x, y$. Аналогично вершины $v_{1}$ и $v_{2}$ не соседние тогда и только тогда, когда в графе $\mathscr{A}$ между $x$ и $y$ есть путь $x-t-y$ такой, что $t<x, y$.

Пусть $\tau: \omega \rightarrow I_{2}-$ биективная эффективная нумерация максимального множества попарно не соседних в $\mathscr{A}=\{A ; E\}$ элементов. С учетом вышеизложенного структура $\mathscr{G}=\left\{\omega, E_{G}\right\}$, где

$$
\begin{aligned}
& E_{G}(x, y) \Leftrightarrow(\exists v)[v>\tau(x) \& v>\tau(y) \& E(x, v) \& E(y, v)], \\
& \neg E_{G}(x, y) \Leftrightarrow(\exists v)[v<\tau(x) \& v<\tau(y) \& E(x, v) \& E(y, v)],
\end{aligned}
$$

будет искомым графом. Таким образом, отношение $E_{G}$ и его дополнение $\neg E_{G}$ перечислимы относительно $\operatorname{deg}(\mathscr{A})$ и, следовательно, $E_{G}$ вычислимо относительно $\operatorname{deg}(\mathscr{A})$. Так как $\operatorname{dg} \operatorname{Sp}(\mathscr{G})$ замкнут наверх, существует представление $\mathscr{G}$ степени $\operatorname{deg}(\mathscr{A})$.

Следующая лемма оправдывает выбранное обозначение $\mathscr{F}^{-1}$.

Лемма 1. Для любого графа $\mathscr{G}$ имеет место $\mathscr{F}^{-1}(\mathscr{F}(\mathscr{G})) \cong \mathscr{G}$.

ДокаЗАтельство. Пусть $\mathscr{G}_{1}=\left\{\omega ; E_{G_{1}}\right\}$ - исходный граф и $\mathscr{G}_{2}=\left\{\omega ; E_{G_{2}}\right\}$ $=\mathscr{F}^{-1}\left(\mathscr{F}\left(\left(\mathscr{G}_{2}\right)\right)\right)$. Имеет место биекция $\varphi: n \mapsto I_{2, n}$ между множеством натуральных чисел и разбиением $I_{2}$. В свою очередь, нумерация $\tau: \omega \rightarrow I_{2}$ определяет биекцию $\psi$ между разбиением $I_{2}$ и $\omega$ следующим образом: $\psi: I_{2, j} \mapsto k$ тогда и только тогда, когда $\tau(k) \in I_{2, j}$. Таким образом, композиция $\varphi \circ \psi: \omega \rightarrow \omega$ является биекцией. Пусть выполнено $E_{G_{1}}(x, y)$, тогда любой элемент $I_{2, x}$ соединен с любым элементом $I_{2, y}$ путем длины 2 , причем так, что центральная вершина больше (в порядке $<$ ) начальной и конечной. В частности, это верно для $\tau(k)$ и $\tau(m)$ таких, что $\tau(k) \in I_{2, x}$ и $\tau(k) \in I_{2, y}$, следовательно, $E_{G_{2}}(k, m)$. Нетрудно видеть, что $\varphi \circ \psi(x)=k$ и $\varphi \circ \psi(y)=m$.

Пусть $\neg E_{G_{1}}(x, y)$. Тогда любой элемент $I_{2, x}$ соединен с любым элементом $I_{2, y}$ путем длины 2 , причем так, что центральная вершина меньше (в порядке 
$<)$ начальной и конечной. В частности, это верно для $\tau(k)$ и $\tau(m)$ таких, что $\tau(k) \in I_{2, x}$ и $\tau(k) \in I_{2, y}$, следовательно, $\neg E_{G_{2}}(k, m)$. Нетрудно видеть, что $\varphi \circ \psi(x)=k$ и $\varphi \circ \psi(y)=m$. Таким образом,

$$
E_{G_{1}}(x, y) \leftrightarrow E_{G_{2}}(\varphi \circ \psi(x), \varphi \circ \psi(y)) .
$$

Лемма 2. Если $\mathscr{G}_{1} \cong \mathscr{G}_{2}$, то $\mathscr{F}\left(\mathscr{G}_{1}\right) \cong \mathscr{F}\left(\mathscr{G}_{2}\right)$.

ДокаЗАТЕЛЬСТво. Пусть $\varphi: \omega \rightarrow \omega-$ изоморфизм графов $\mathscr{G}_{1}, \mathscr{G}_{2}$ и $\mathscr{F}\left(\mathscr{G}_{1}\right)=\left\{A_{1} ;<_{1}, E_{1}\right\}, \mathscr{F}\left(\mathscr{G}_{2}\right)=\left\{A_{2} ;<_{2}, E_{2}\right\}$.

Рассмотрим отображение $\nu_{1}^{-1} \circ \varphi \circ \nu_{2}$. Оно является биекцией между разбиениями $\left\{I_{1, j}\right\}$ и $\left\{I_{1, j}^{\prime}\right\}$. Аналогично $\mu_{1}^{-1} \circ \varphi \circ \mu_{2}-$ биекция между разбиениями $I_{3, j}$ и $\left\{I_{3, j}^{\prime}\right\}$.

Определим следующие отображения:

1) $f_{1, j}$, действующие из $I_{1, j}$ в $\left\{I_{1, \nu_{1}^{-1} \circ \varphi^{\prime} \nu_{2}(j)}^{\prime}\right\}$,

2) $f_{2, j}$, действующие из $I_{2, j}$ в $\left\{I_{2, \varphi(j)}^{\prime}\right\}$,

3) $f_{3, j}$, действующие из $I_{3, j}$ в $\left\{I_{3, \mu_{1}^{-1} \circ \varphi_{\circ}(j)}^{\prime}\right\}$.

Для любого $j$ существует изоморфизм $\tau_{i, j}: I_{i, j} \rightarrow I_{1, \nu_{1}^{-1} \circ{ }^{\prime} \circ \nu_{2}(j)}^{\prime}$, так как оба множества изоморфны $\mathbb{Q}$. При определении $f_{i, j}$ нужно добиться того, что изоморфизм $f_{i, n+1}$ должен быть согласован с $\bigcup_{j \leq n} f(i, j)$, т. е. определен так, чтобы функция $\bigcup_{j \leq n} f_{i, j}$ была изоморфизмом между $\bigcup_{j \leq n} I_{i, j}$ и $\bigcup_{j \leq n} I_{1, \nu_{1}^{-1} \circ \varphi^{\prime} \circ \nu_{2}(j)}^{\prime}$. При произвольном выборе изоморфизмов $f_{i, j}, 0 \leq j \leq n$, такого $f_{i, n+1}$ может вообще не существовать. Однако существование согласованных семейств изоморфизмов $\left\{f_{i, j}\right\}_{j=0}^{\infty}, i=1,2,3$, легко следует из стандартной челночной конструкции и плотностей $I_{i, j}$ в $I_{i}$ и $I_{i, j}^{\prime}$ в $I_{i}^{\prime}$ соответственно.

Коротко дадим схему доказательства данного факта. Для каждого $j$ рассмотрим следующие требования:

$R_{i, j}$ - отображение $f(i, j)$ является всюду определенным на $I_{i, j}$ сохраняющим порядок инъективным отображением в $I_{1, \nu_{1}^{-1} \circ \varphi_{\nu}(j)}^{\prime}$, согласованным c $f(i, k), k \neq j$;

$M_{i, j}$ - отображение $f(i, j)$ является сюръективным сохраняющим порядок инъективным отображением $I_{i, j}$ на $I_{1, \nu_{1}^{-1} \circ \varphi_{\circ} \nu_{2}(j)}^{\prime}$, согласованным с $f(i, k), k \neq j$.

Считаем, что каждое рассматриваемое бесконечное множество имеет нумерацию натуральными числами. Конструкция проводится по шагам. На шаге $s$ работают требования с номерами $j \leq s$. Требование вида $R_{i, j}$ находит элемент $x$ из $I_{i, j}$ с наименьшим номером, для которого значение $f_{i, j}(x)$ еще не определено, далее находит элемент $y$ из $I_{1, \nu_{1}^{-1} \circ \varphi_{\circ}(j)}^{\prime}$ с наименьшим номером такой, что определение $f_{i, j}(x)=y$ будет согласовано со всеми уже определенными частичными изоморфизмами. Требование вида $M_{i, j}$ выбирает элемент $y$ из $I_{1, \nu_{1}^{-1} \circ{\text { о } \nu_{2}(j)}^{\prime}}^{\prime}$ с наименьшим номером, для которого прообраз относительно $f_{i, j}$ еще не определен, далее находит элемент $x$ из $I_{i, j}$ с наименьшим номером, для которого определение $f_{i, j}(x)=y$ будет согласовано со всеми уже определенными частичными изоморфизмами. Для обоих требований искомый элемент существует в силу плотностей $I_{i, j}$ в $I_{i}$ и $I_{i, j}^{\prime}$ в $I_{i}^{\prime}$. Остальные детали оставляем читателю. 
Итак, имеем

$$
\begin{gathered}
\left(\forall x<y \in \bigcup_{j \leq n} I_{i, j}\right)\left(\exists z \in I_{i, n+1}\right)[x<z<y], \\
\left(\forall x<y \in I_{i, n+1}\right)\left(\exists z \in \bigcup_{j \leq n} I_{i, j}\right)[x<z<y] ; \\
\left(\forall x<y \in \bigcup_{j \leq n} I_{1, \nu_{1}^{-1} \circ \varphi \circ \nu_{2}(j)}^{\prime}\right)\left(\exists z \in I_{1, \nu_{1}^{-1} \circ \varphi \circ \nu_{2}(n+1)}^{\prime}\right)[x<z<y], \\
\left(\forall x<y \in I_{1, \nu_{1}^{-1} \circ \varphi \circ \nu_{2}(n+1)}^{\prime}\right)\left(\exists z \in \bigcup_{j \leq n} I_{1, \nu_{1}^{-1} \circ \varphi \circ \nu_{2}(j)}^{\prime}\right)[x<z<y] .
\end{gathered}
$$

Нетрудно видеть, что построенная таким образом функция $f=\bigcup_{i=1}^{3} \bigcup_{j \in \omega} f_{i, j}$ является изоморфизмом линейных порядков $\left\{A_{1} ;<_{1}\right\}$ и $\left\{A_{2} ;<_{2}\right\}$. Осталось показать, что $f$ является изоморфизмом структур $\mathscr{F}\left(\mathscr{G}_{1}\right)=\left\{A_{1} ;<_{1}, E_{1}\right\}$ и $\mathscr{F}\left(\mathscr{G}_{1}\right)=$ $\left\{A_{2} ;<_{2}, E_{2}\right\}$.

Для $x, y \in A_{1}$ выполняется $E_{1}(x, y)$ тогда и только тогда, когда имеет место один из следующих случаев.

Случай 1. Пусть $(\exists k)\left[x, y \in I_{2, k}\right]$. В этом случае $f(x), f(y) \in I_{2, \varphi(k)}^{\prime}$ и, следовательно, $E_{2}(f(x), f(y))$.

СлучАй 2. Пусть $(\exists k)(\exists m)\left[x \in I_{2, k} \& y \in I_{1, m}\right]$. Это возможно в том случае, если в $G_{1}$ существует ребро $e_{1}$ такое, что $k \in e_{1}$ и $\nu_{1}\left(e_{1}\right)=m$. Тогда $\varphi(x) \in \varphi\left(e_{1}\right)$ и $\nu_{2}\left(\varphi\left(e_{1}\right)\right)=\nu_{1}^{-1} \circ \varphi \circ \nu_{2}(m)$. Так как $f(x) \in I_{2, \varphi(k)}^{\prime}$ и $f(y) \in I_{1, \nu_{1}^{-1} \circ \varphi \circ \nu_{2}(m)}^{\prime}$, то $E_{2}(f(x), f(y))$.

Случай 3. Пусть $(\exists k)(\exists m)\left[x \in I_{2, k} \& y \in I_{3, m}\right]$. Это возможно в том случае, если в $G_{1}$ существует вершина $v$ такая, что $\neg E_{G_{1}}(k, v)$ и $\mu_{1}(\{k, v\})=m$. Тогда $\neg E_{G_{2}}(\varphi(k), \varphi(v))$ и $\nu_{2}(\{\varphi(k), \varphi(v)\})=\mu_{1}^{-1} \circ \varphi \circ \mu_{2}(m)$. Так как $f(x) \in I_{2, \varphi(k)}^{\prime}$ и $f(y) \in I_{3, \mu_{1}^{-1} \circ \varphi \circ \mu_{2}(m)}^{\prime}$, то $E_{2}(f(x), f(y))$.

Нетрудно видеть, что $f$ вычислим относительно $\mathscr{G}_{1} \oplus \varphi \oplus \mathscr{G}_{2}$.

Естественной модификацией доказательств легко получить леммы, аналогичные леммам 1, 2 и справедливые для произвольных морфизмов, а не только для изоморфизмов.

ЗАмЕчАНИЕ. Использование элементов $q_{1}$ и $q_{2}$ делает конструкцию теоремы неравномерной. Отметим, что этого можно избежать. Например, можно определить на $q_{1}$ и $q_{2}$ петли либо закодировать в качестве разделителей конечные куски, изоморфные $K_{5}\left(K_{5}\right.$ - полный граф с пятью вершинами), которые в основной конструкции не встречаются.

Как отмечено выше, существование вычислимого функтора позволяет транслировать различные алгоритмические свойства из одной категории структур в другую [8]. В частности, инъективный вычислимо обратимый вычислимый функтор сохраняет спектры степеней алгебраических структур, и из спектральной универсальности графов получаем

Следствие. Класс структур вида $\mathscr{A}=\{\mathbb{Q} ;<, E\}$ спектрально универсален. 


\section{ЛИТЕРАТУРА}

1. Hirschfeldt D. R., Khoussainov B., Shore R. A., Slinko A. M. Degree spectra and computable dimensions in algebraic structures // Ann. Pure Appl. Logic. 2002. V. 115, N 1-3. P. 71-113.

2. Калимуллин И. Ш., Селиванов В. Л., Фролов А. Н. Спектры степеней структур // Итоги науки и техники. Современная математика и ее приложения Тематические обзоры. Т. 158. М.: ВИНИТИ РАН, 2018. С. 23-39.

3. Harizanov V., Miller R. Spectra of structures and relations // J. Symb. Log. 2007. V. 72 , N 1. P. 324-348.

4. Richter L. J. Degrees of structures // J. Symb. Log. 1981. V. 46, N 4. P. 723-731.

5. Miller R. The $\Delta_{2}^{0}$-spectrum of a linear order // J. Symb. Log. 2001. V. 62, N 2. P. 470-486.

6. Фролов $A$. H. Заметка о $\Delta_{2}^{0}$-спектрах линейных порядков и спектрах отношения соседства на них // Изв. вузов. Математика. 2013. № 11. С. 74-78.

7. Frolov A., Kalimullin I., Harizanov V., Kudinov O., Miller R. Spectra of high $\mathrm{n}$ and non-low n degrees // J. Logic Comput. 2010. V. 22, N 4. P. 755-777.

8. Miller R., Poonen B., Schoutens H., Shlapentokh A. A computable functor from graphs to fields // J. Symb. Log. 2018. V. 83, N 1. P. 326-349.

9. Harrison-Trainor M., Melnikov A., Miller R., Montalban A. Computable functors and effective interpretability // J. Symb. Log. 2018. V. 82, N 1. P. 77-97.

10. Баженов Н. А., Фролов А. Н., Калимуллин И. Ш., Мельников А. Г. Вычислимость дистрибутивных решеток // Сиб. мат. журн. 2017. Т. 58, № 6. С. 1236-1251.

Поступила в редакцию 25 декабря 2018 г.

После доработки 10 января 2020 г.

Принята к публикачии 8 апреля 2020 г.

Зубков Максим Витальевич

Казанский (Приволжский) федеральный университет,

ИММ им. Н. И. Лобачевского,

ул. Кремлевская, 18, Казань 420008

maxim.zubkov@kpfu.ru

Фролов Андрей Николаевич

Казанский (Приволжский) федеральный университет,

ВШ ИТиС,

ул. Кремлевская, 18, Казань 420008

a.frolov.kpfu@gmail.com 